\title{
Investigation of Band Tailing in Sputtered ZnO:Al Thin Films Regarding Structural Properties and Impurities
}

\author{
Steffi Schönau ${ }^{1}$, Florian Ruske ${ }^{1}$, Sebastian Neubert ${ }^{2}$, Bernd Rech $^{1}$ \\ ${ }^{1}$ Helmholtz-Zentrum Berlin für Materialien und Energie GmbH, Institute for Silicon \\ Photovoltaics, Kekuléstr. 5, 12489 Berlin \\ ${ }^{2}$ Helmholtz-Zentrum Berlin für Materialien und Energie GmbH, PVcomB, Schwarzschildstr. 3, \\ 12489 Berlin
}

\begin{abstract}
Thin films of pure aluminum doped $\mathrm{ZnO}$ and with addition of nitrogen, oxygen and hydrogen have been prepared by magnetron sputtering. The spectral absorption coefficient close to the band gap energy has been determined by spectrophotometry and analyzed regarding band tailing and creation of defect bands. We found, that an improvement of Raman crystallinity under $\mathrm{O}_{2-}$ rich growth conditions is not accompanied by a suppression of band tailing as expected. An additional absorption feature evolves for layers grown in $\mathrm{N}_{2}$ containing atmosphere. Doping with hydrogen attenuates sub-band gap absorption.
\end{abstract}

\section{INTRODUCTION}

Doped zinc oxide is a key material in various optoelectronic devices. A suitable way to adjust electrical and optical properties, at least for sputtered, doped $\mathrm{ZnO}$, is the application of thermal post-deposition treatments including at least one step where the $\mathrm{ZnO}: \mathrm{Al}$ is protected by a thin protective layer. This method leads not just to a considerably enhanced mobility but to significantly reduced sub-band gap absorption, as well ${ }^{1,2,3}$. However, although absorption tails below the band gap are well known for $\mathrm{ZnO}$ :Al prepared by various deposition techniques, a deep understanding of the underlying mechanisms is still missing.

In an earlier work we studied the appearance of such tails in non-reactively sputtered $\mathrm{ZnO}: \mathrm{Al}$ thin films in respect to deposition conditions and applied annealing treatments ${ }^{3}$. In this work special emphasis was put on the influence of impurities on the Urbach energy $E_{U}$. It is usually assumed, that optical transmission is heavily influenced by film stoichiometry and that increasing deposition temperature has a beneficial effect on transmittance due to the evaporation of excess zinc. Here, we therefore studied the influence of oxygen addition during the deposition process on band tailing, electrical properties and Raman activity of the $\mathrm{ZnO}: \mathrm{Al}$.

Nitrogen on the other hand is always present in small quantities in production sputtering coaters and is known to lead to a yellowish to brownish coloring of $\mathrm{ZnO}$ when purposely added to the process. Therefore, nitrogen addition was investigated for various deposition conditions and post-deposition treatments.

Finally, it was investigated whether a passivation of optically active defects can be reached by addition of hydrogen to the process.

\section{EXPERIMENTAL DETAILS}


The $\mathrm{ZnO}$ :Al thin films were deposited on $1.1 \mathrm{~mm}$ thick Corning Eagle XG glass substrates with a dimension of $100 \mathrm{~mm} \times 100 \mathrm{~mm}$ using rf-magnetron sputtering from a circular ceramic target of $250 \mathrm{~mm}$ in diameter and $1 \% \mathrm{wt}$. aluminum oxide. The target to substrate distance was fixed to $124 \mathrm{~mm}$ and the process was carried out in pure $\mathrm{Ar}$ or in $\mathrm{Ar}+\mathrm{X}$, where $\mathrm{X}=\mathrm{N}_{2}, \mathrm{O}_{2}, \mathrm{H}_{2}$, sputtering gas mixture. The total chamber pressure and rf-power were set to $250 \mathrm{mPa}$ and $750 \mathrm{~W}$, respectively. The maximum addition of reactive gas was $2 \%\left(\mathrm{O}_{2}\right), 2.5 \%\left(\mathrm{~N}_{2}\right)$ and $5 \%\left(\mathrm{H}_{2}\right)$. Deposition was carried out at $200{ }^{\circ} \mathrm{C}$ in the case of oxygen and nitrogen addition and at $100{ }^{\circ} \mathrm{C}$ for hydrogen addition.

After deposition, parts of all samples were thermally treated (a) for 24 hours at $550{ }^{\circ} \mathrm{C}$ without any capping (degradation) and (b) for 24 hours at $650{ }^{\circ} \mathrm{C}$ under a protective $50 \mathrm{~nm}$ a-Si layer (cap annealing). All annealing processes were carried out under nitrogen flow and atmospheric pressure. After annealing, the a-Si cap was removed using a $\mathrm{NF}_{3}$ plasma etch step. A detailed description of the annealing procedures can be found elsewhere ${ }^{1,3}$.

Optical properties were analyzed via reflection and transmission spectra. From these, the absorption coefficient $\alpha$ of the film was calculated and analyzed around the band gap energy. For this we used a combined expression consisting of an error function to describe $\alpha$ for energies above the band gap and an Urbach expression for lower energies. Details of the procedure can be found elsewhere ${ }^{3}$.

\section{RESULTS}

\section{A. Prior results for varying deposition temperature}

In an earlier work, we found that the deposition temperature and post-deposition thermal treatments have a tremendous influence on the appearance of band tails ${ }^{3}$. It was found, that $\mathrm{ZnO}: \mathrm{Al}$ thin films deposited at low temperatures are characterized by a high Urbach energy and a rather low resistivity. Increasing the deposition temperature reduces $E_{U}$ but leads to higher resistivity at temperatures above $400{ }^{\circ} \mathrm{C}$. The latter is caused by a decrease in doping efficiency and an accompanied reduction of the mobility.

Application of thermal post-deposition treatments, both, with and without protective a-Si cap, results in significantly less band tailing, just weakly dependent on the deposition conditions. However, in contrast to annealing without cap (degradation), applying cap annealing leads to the more desirable thin films with low resistivity and a low sub-band gap absorption caused by band tailing. This has shown that band tailing is not connected to the electrical properties of the samples. Raman spectroscopy, on the other hand, showed that the deposition temperature and post-deposition annealing has a significant influence on the lattice disorder and a correlation of structural quality and Urbach energy could be assumed ${ }^{4}$.

\section{B. Influence of gas composition B.1. Electrical properties}

Adding oxygen or nitrogen to the sputtering gas results in a strong increase of resistivity (fig. 1). For nitrogen addition, the highest nitrogen flows resulted in a resistivity increase in comparison to the sample deposited in pure Ar by more than five orders of magnitude.

Degradation of films deposited under oxygen addition (fig. 1a) results in resistivities in the order of $1 \Omega \mathrm{cm}$, with only little influence of the oxygen flow during deposition. Cap annealing on the other hand results in strongly reduced resistivities, again with only small influences of oxygen 


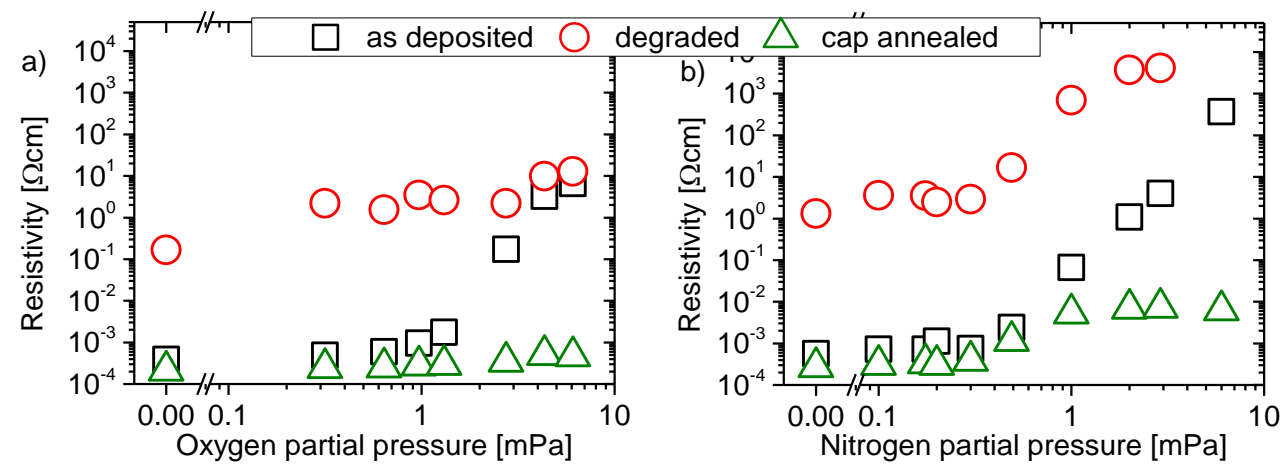

Figure 1. Resistivity as function of the a) oxygen and b) nitrogen partial pressure used for deposition.

flow during deposition. Thus annealing procedures seem to mostly remove the effect of oxygen addition during growth on electrical properties.

For nitrogen addition (fig. 1b) the general trend of resistivity increase by degradation and decrease for cap anneal is the same. But in contrast to the oxygen addition, the results are more dependent on nitrogen content and significantly higher resistivities are obtained both after degradation and cap anneal for samples deposited under high nitrogen flows.

It should be noted, that annealing of the degraded samples was carried out under flowing nitrogen for both sample series, but the resulting electrical properties vary in between samples deposited with oxygen or nitrogen addition. Apparently nitrogen can only be incorporated during deposition, but already incorporated nitrogen can lead to the activation of acceptor-like defects during thermal treatment.

\section{B.2. Influence of oxygen addition on band-tailing and structural properties}

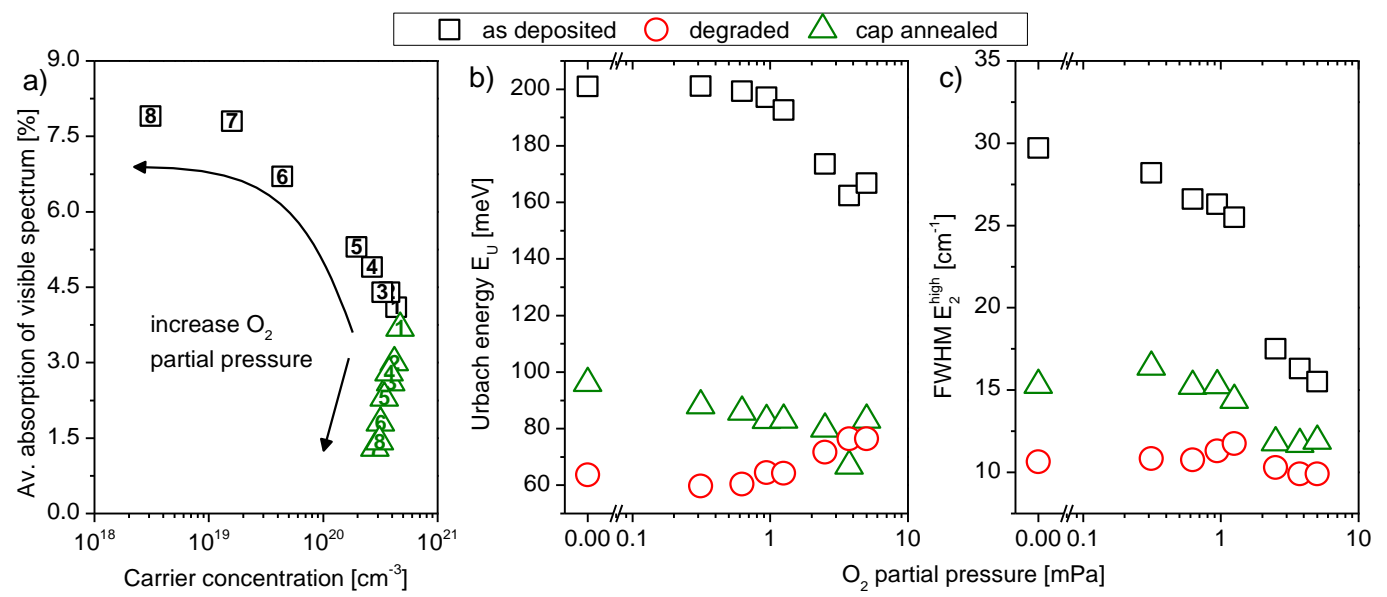

Figure 2. a) Average absorption of the layers in the range of $380-780 \mathrm{~nm}$ as a function of carrier concentration for as deposited and cap annealed $\mathrm{ZnO}$ :Al thin films. The rising numbers represent an increase in $\mathrm{O}_{2}$ partial pressure. b) shows Urbach energy and c) FWHM of the Raman $\mathrm{E}_{2}$ (high) resonance as a function of oxygen partial pressure for the as deposited and cap annealed state. All layers have a thickness of [700 \pm 12$] \mathrm{nm}$. 
The additional supply of oxygen during growth should change the films stoichiometry and alters both electrical and optical properties significantly. Due to the decrease in carrier concentration, the band gap shifts to lower energies ${ }^{5}$, while the Urbach energy of the absorption tails only drops slightly (fig 2 b). The reduced band gap results in the shift of the still strong absorption tails into the visible region of the spectrum and causes more absorption of blue radiation, resulting in a lowered visual transmission (fig 2 a).

For the cap annealed samples, the overall lower visible absorption is attributed to the higher carrier concentration, corresponding to a band gap far in the UV, and a contraction of the band tails. The further decrease of absorption with increasing oxygen concentration is directly linked to the decrease in $E_{U}$.

The supply of additional oxygen during growth and the application of thermal post-deposition treatments also have a strong influence on the Raman spectra (not shown here). As can be already seen in fig. $2 \mathrm{c}$, degradation of the films results in the lowest lattice disorder, quantified by the FWHM of the intrinsic $\mathrm{E}_{2}$ (high) mode at $v=439 \mathrm{~cm}^{-1}$. For as deposited films we observed an improvement of Raman crystallinity with strong oxygen addition, but in contrast to results obtained for deposition at different temperatures ${ }^{3}$, this is not accompanied by a strong reduction in $E_{U}$. So obviously, a better Raman crystallinity is not sufficient to exclude band tailing.

Additionally to backscattering active resonances expected in c-axis oriented $\mathrm{ZnO}^{6,7}$, additional modes evolve. We noticed, that modes at $v=275$ and $v=510 \mathrm{~cm}^{-1}$ always occur in pairs and are suppressed above a critical $\mathrm{O}_{2}$ concentration, both in the as deposited, as well as for both thermal post-deposition treatments. The origin of these modes is discussed controversially in literature. The most common explanations attribute these resonances to disorder-activated Raman silent Bmodes by a break of lattice symmetry, caused by dopants or growth conditions ${ }^{8-10}$. Additionally, there are studies ascribing the existence of the mode at $v=275 \mathrm{~cm}^{-1}$ to the presence of interstitial $\mathrm{Zn}\left[\mathrm{Zn}_{\mathrm{i}}\right]$, acting as shallow donor ${ }^{11,12}$. A pronounced phonon mode at $\mathrm{v}=577 \mathrm{~cm}^{-1}$, assigned to resonant enhanced $\mathrm{E}_{1}$ and $\mathrm{A}_{1}(\mathrm{LO})$ phonons in $\mathrm{ZnO}$, caused by localized states in the band gap ${ }^{13}$, vanishes completely after cap annealing but not after degrading, for samples prepared with more than $0.1 \% \mathrm{O}_{2}$ in deposition atmosphere. However, no obvious correlation between the development of the resonance at $577 \mathrm{~cm}^{-1}$ and the formation of optically active band tails can be observed.

\section{B.3. Influence of nitrogen addition on band tailing}

The absorption coefficient of films grown with nitrogen addition exhibits a pronounced feature around $2.8 \mathrm{eV}$ which cannot be fitted with a simple Urbach tail. We therefore added a Gaussian to model the nitrogen related absorption. As can be seen in figure 3 a) this extension to our model agrees very well with the experimental data. The extracted parameters like Urbach energy $E_{U}$ and the area of the fitted gauss peak are visualized in figure $3 \mathrm{~b}$ ) and c), respectively. Due to the strong overlap of the nitrogen related absorption and the Urbach tail, a separation of both contributions is not easy and minor changes could arise if, for instance, an asymmetric function was used to model nitrogen related absorption.

As can be seen in figure $3 \mathrm{c}$ ) the absorption peak around $2.8 \mathrm{eV}$ correlates very strongly with the $\mathrm{N}_{2}$ concentration during growth. Assuming, that the area of that peak is directly related to the $\mathrm{N}_{2}$ content in the sample, it is interesting to note, that neither cap annealing nor degradation have 

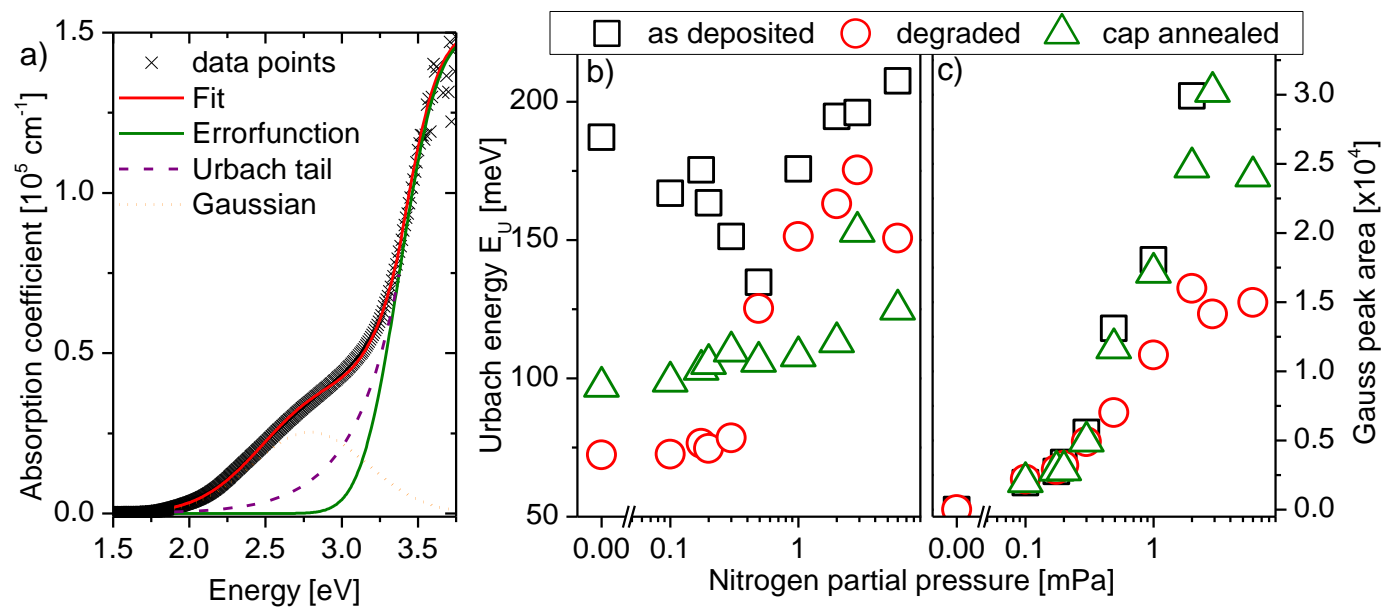

Figure 3. a) Example of the fitting routine including the addition of a gauss function to allow for the nitrogen absorption; b) results for the fitting parameter Urbach energy and c) area of the Gaussian in dependence on the nitrogen content during growth and for all thermal postdeposition treatments.

the ability to remove nitrogen completely but a specific equilibrium level could be assumed for annealing without protective layer.

For the Urbach energy derived with our model, the data scatters significantly. In the asdeposited state, a general trend of increasing $E_{U}$ with nitrogen addition can be assumed. More interestingly, cap annealing leads to strongly reduced $E_{U}$ for all samples, showing that the Urbach energy is mostly a different effect to the nitrogen-related absorption described by the Gaussian. Degradation finally also reduces the Urbach energy, but a strong influence of the nitrogen content in the films remains.

Nitrogen addition also has a tremendous effect of Raman spectra (not shown here). Already small additions of nitrogen lead to strong modes at 275 and $577 \mathrm{~cm}^{-1}$. The $\mathrm{E}_{2}$ (high) mode is supressed and a shift of the modes towards lower wavenumbers is observed for strong nitrogen additions. While a full picture has not been derived yet, a strong influence of nitrogen on film growth is obvious.

In summary it can be concluded, that nitrogen as an impurity in $\mathrm{ZnO}$ causes considerable subband gap absorption, but thermal treatments only partially remove this effect. As thermal annealing of films deposited in pure Argon removes band tailing almost completely, the contribution of residual nitrogen to this absorption is expected to be small.

\section{B.4. Influence of hydrogen supply during growth}

For addition of hydrogen the deposition temperature was switched to $100{ }^{\circ} \mathrm{C}$. At this temperature we were able to get smooth films and a clear influence of the added hydrogen. Regarding electrical properties, we were able to reproduce the increase of carrier concentration with increasing hydrogen as reposted in numerous papers ${ }^{14}$. Our main interest was in sub-band gap absorption and the determination of $E_{U}$.

Hydrogen has an immediate influence on the band tailing and a steady decrease of Urbach energy with hydrogen flow can be observed down to a saturation level around $120 \mathrm{meV}$. Up to now, the reason is yet unknown, but a passivation of the tail-causing defects seems possible. Low 
temperature anneals to drive out the hydrogen from the sample are currently being carried out and could give hints, if the decrease in $E_{U}$ is related to the presence of hydrogen in the films or to a different growth of the films.

\section{CONCLUSIONS}

In conclusion, the addition of impurities to the growth process of $\mathrm{ZnO}$ : $\mathrm{Al}$ can have severe influences on both electrical and optical properties. Both, the addition of $\mathrm{O}_{2}$ and $\mathrm{N}_{2}$ increases the resistivity of sputter deposited $\mathrm{ZnO}: \mathrm{Al}$ strongly. We observed that thermal post-deposition treatment mostly removes the influence of $\mathrm{O}_{2}$ but not of $\mathrm{N}_{2}$ on the resistivity.

In contrast to earlier results, we found that pronounced band tailing occurs even in material with improved crystallinity if samples are deposited under oxygen rich conditions. $\mathrm{ZnO}: \mathrm{Al}$ films grown in $\mathrm{N}_{2}$-containing atmosphere are characterized by additional absorption close to the band edge, which cannot be described by Urbach band tailing alone. This $\mathrm{N}_{2}$-related absorption is not or only partially removable by extended thermal treatments.

Additionally, we could show that hydrogen efficiently prevents pronounced band tailing in the as deposited state.

\section{ACKNOWLEDGMENTS}

We thank Martin Reiche for technical assistance, Marc A. Gluba for support with the Raman setup and Martina Trahms for performing optical and Hall spectroscopy. This work was supported by the Federal Ministry for the Environment, Nature Conservation and Nuclear Safety BMU under contract No. 0325299E.

\section{REFERENCES}

1. F. Ruske, M. Roczen, K. Lee, M. Wimmer, S. Gall, J. Hüpkes, D. Hrunski, and B. Rech, Journal of Applied Physics 107, 013708 (2010).

2. S. Neubert, M. Wimmer, F. Ruske, S. Calnan, O. Gabriel, B. Stannowski, R. Schlatmann, and B. Rech, Prog. Photovoltaics (2013, published online).

3. S. Schönau, F. Ruske, S. Neubert, and B. Rech, Appl. Phys. Lett. 103, 192108 (2013).

4. S. Schönau, F. Ruske, S. Neubert, and B. Rech, Phys. Status Solidi C (2013, to be published).

5. A.P. Roth, J.B. Webb and D.F. Williams, Thin Solid Films 349, 1-2, p. 93-99 (1999) .

6. T. C. Damen, S. P. S. Porto, and B. Tell, Physical Review 142(2), 570-574 (1966).

7. C. A. Arguello, D. L. Rousseau, and S. P. S. Porto, Phys. Rev. 181, 1351-1363 (1969).

8. C. Bundesmann, N. Ashkenov, M. Schubert, D. Spemann, T. Butz, E. M. Kaidashev, M.

Lorenz, and M. Grundmann, Applied Physics Letters 83(10), 1974 (2003).

9. F. J. Manjón, B. Marí, J. Serrano, Romero, and A. H. Romero, Journal of Applied Physics 97(5), 053516 (2005).

10. M. Tzolov, N. Tzenov, D. Dimova-Malinovska, M. Kalitzova, and C. Pizzuto, Thin Solid Films 379, 28-36 (2000).

11. F. Friedrich, M. A. Gluba, and N. H. Nickel, Applied Physics Letters 95(14), 141903 (2009).

12. M. A. Gluba, N. H. Nickel, and N. Karpensky, Phys. Rev. B 88, 245201 (2013).

13. F. Friedrich and N. H. Nickel, Appl. Phys. Lett. 91, 111903 (2007).

14. see for example: M.L. Addonizio, A. Antonaia, G. Cantele, C. Privato, Thin Solid Films 349, 93-99 (1999). 